\title{
A methodology utilizing continuous source and receiver side wavefields
}

\author{
Stian Hegna, Tilman Klüver and Jostein Lima*, PGS
}

Copyright 2019, SBGf - Sociedade Brasileira de Geofísica

This paper was prepared for presentation during the $16^{\text {th }}$ International Congress of the Brazilian Geophysical Society held in Rio de Janeiro, Brazil, 19-22 August 2019.

Contents of this paper were reviewed by the Technical Committee of the $16^{\text {th }}$ International Congress of the Brazilian Geophysical Society and do not necessarily represent any position of the SBGf, its officers or members. Electronic reproduction or storage of any part of this paper for commercial purposes without the written consent of the Brazilian Geophysical Society is prohibited.

\section{Abstract}

We describe a novel acquisition and processing method which makes use of continuous wavefields on both the source and receiver side to extract the response of the earth from marine seismic data. The methodology is called "eSeismic". Changes to the typical marine seismic acquisition method and the processing methodology will be outlined. The benefits gained from working with continuous wavefields and denser source side sampling will be discussed, in addition to improved acquisition efficiency and reduced environmental footprint by emitting lower sound pressure levels.

\section{Introduction}

Traditionally in marine seismic acquisition, several airguns in arrays are typically triggered simultaneously at pre-defined positions. Seismic data are recorded for as long as necessary to be able to generate an image of the sub-surface to a desired depth. One seismic shot record is associated with each source array activation, and it is assumed that each record does not contain energy from previous source activations. Hence, residual energy from previous shots will end up as noise. This is commonly referred to as shot-generated noise. The air-gun arrays are tuned to emit a wavefield with a wavelet as close as possible to a band-limited spike. The listening time required to achieve the imaging goals of a survey defines the distance between consecutive shot points. A typical example is $25 \mathrm{~m}$ shot point interval allowing for $10 \mathrm{~s}$ listening time at a maximum bottom speed of $2.5 \mathrm{~m} / \mathrm{s}(4.86$ knots). With typical dual source acquisition, this results in $50 \mathrm{~m}$ spacing between shots on each source line. This gives significantly coarser sampling than the typical $12.5 \mathrm{~m}$ channel spacing available on the receiver side.

Various acquisition and processing methods have been proposed in recent years that employ blended or simultaneous source techniques in order to improve the spatial sampling on the source side or increase acquisition efficiency (e.g. BEASLEY et al., 1998; BERKHOUT, 2008; FRØMYR et al., 2008; ROBERTSSON et al. 2016; SJØEN PEDERSEN et al., 2016). However, all these methods use the concept of discrete shot records. When the energy from several blended source activations is overlapping, 'de-blending' techniques are used to create individual records in positions associated with each of the blended sources. The methods referenced above make use of similar tuned air-gun arrays already described. The wavefields emitted from the blended sources are therefore highly correlated because the wavefield emitted from each source has a wavelet approaching a band-limited spike. This makes it challenging to separate the wavefields associated with each source array.

Methods using encoded source sequences (e.g. ABMA and ROSS, 2013; MUELLER et al., 2014) reduce the similarity between the signals emitted by multiple sources simultaneously. The coding in the sequences can be chosen such that the correlation between the signals emitted from multiple sources is limited. These methods also allow for a reduction of the emitted sound pressure levels as the emitted energy is spread out in time instead of being focused in a short bandlimited spike leading to a reduction in the environmental footprint. These methods all assume discrete shot records.

In this paper, we describe a novel acquisition and processing method that is based on continuous wavefields on both the source and the receiver side as illustrated in Figure 1. The method was first introduced in HEGNA et al. (2018) and KLÜVER et al. (2018).
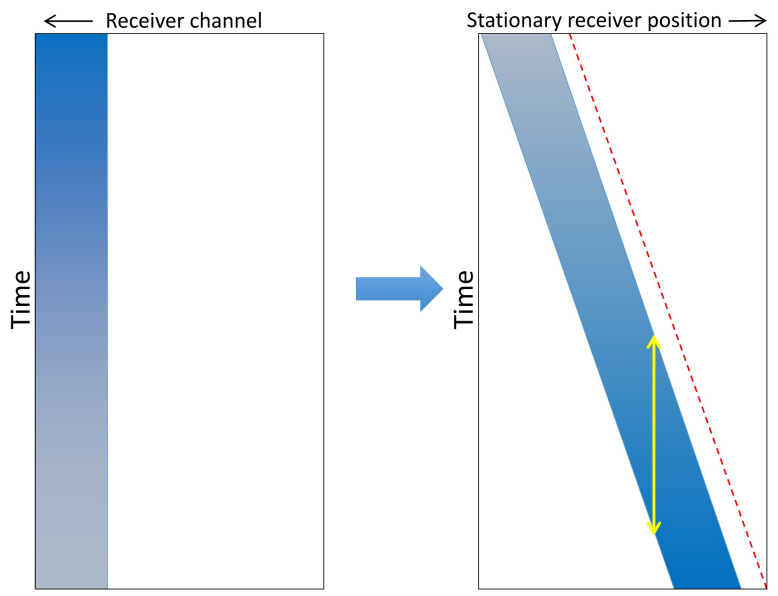

Figure 1 - The blue area to the left illustrates a seismic data record recorded continuously, with a temporal extent of a sail line, and a lateral extent corresponding to the streamer length. The blue area to the right represents the seismic data after receiver motion correction. The spatial extent of these data is the length of the sail line plus the streamer length. The red dashed line indicates the position of a source in front of the streamer as a function of time, and the yellow line represents the live data in a stationary receiver position with a temporal length of the streamer length divided by vessel speed. 
The method allows for a source that is constantly emitting energy while moving. There is no concept of minimum listening time, or no 'shot' records associated with specific 'shot points'. Source energy is spread out in time to reduce the environmental imprint. The movement of all insea equipment is taken into account in the processing of the data. In the case of towed streamer, the received data are placed into the positions where they were received as a function of time. If the data is recorded by stationary receivers, this step is not necessary. The emitted source wavefield is deconvolved from the stationary receiver traces in a multi-dimensional fashion to generate receiver gathers containing the response of the earth, i.e. the wavefield being recorded in that receiver position due to point sources emitting a band-limited spike. In order to enable a stable deconvolution of the emitted wavefield from the received wavefield, the spectrum of the source wavefield needs to be as white as possible both in a temporal and spatial sense. Source elements in multiple cross-line positions can be operated simultaneously and deconvolved into common receiver gathers in the crossline positions because the wavefield emitted can be designed such that it is spatially white also in the crossline direction.

Data recorded over the length of a sail-line is treated continuously during pre-processing. In the following sections we describe key processing steps and discuss the benefits from working with continuous source and receiver side wavefields in terms of improved source side sampling and reduced environmental imprint.

\section{Method}

With today's recording systems on seismic vessels, data are typically recorded continuously for the length of a sailline. In the proposed method we correct for sensor responses and apply noise attenuation on the continuous records to ensure that the continuity of the data is maintained. After these pre-conditioning steps, the lateral motion of the receivers is taken into account by placing the received data into the positions where they were received as a function of time, as illustrated in Figure 1. The data is put into the received positions with a linear phase shift in the wavenumber-time domain.

After the receiver motion correction, the recorded pressure and particle motion data are separated into upgoing and down-going parts. The methodology is as outlined in CARLSON et al. (2007), but applied to the data of one sail-line at once, i.e. to the data as illustrated in the right-hand part of Figure 1, not on a shot record by shot record basis. If streamer depth variations need to be corrected for in a redatuming step, these depth variations are also handled in a continuous fashion.

In the last step specific to the continuous wavefields method, the source wavefield is deconvolved from the stationary receiver trace. We use a multi-dimensional convolutional model to express the data in a stationary receiver location. The entire wavefield that can contribute to a receiver location is expressed as one large wavefield, and the forward modelling of the data received in a stationary position can be expressed as a multi- dimensional convolution of the source wavefield with the earth response. There is a sum over all source emission angles since all emitted energy is recorded in a single receiver location as illustrated in Figure 2.

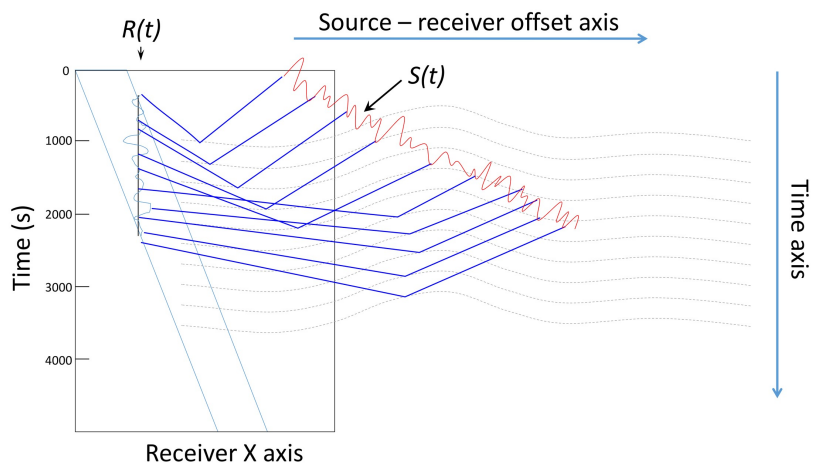

Figure 2 - $R(t)$ is a stationary receiver trace as a function of time, and $S(t)$ are the source signals emitted as a function of time in different offsets relative to the receiver location. The grey dashed lines represent one reflector in the sub-surface, and the blue lines represent some of the ray-paths from the source reflected at the sub-surface reflector and received in the stationary receiver position.

In order to deconvolve the source wavefield from the receiver wavefield, an inverse solution to the forward modelling has to be found. We solve this problem iteratively. Since the source emission angle in a single receiver location is unknown, the received energy is placed in all possible angles and the source wavefield is deconvolved. This guarantees that the signal associated with the response of the earth is focused in the correct locations. However, a lot of energy is placed in incorrect angles generating random noise. Coherent energy is extracted from the deconvolution result and its contribution to the receiver trace is modelled and subtracted to generate a residual receiver trace containing signals that have not yet been explained. The process starts again with deconvolving the source wavefield from the residual receiver trace. In each iteration the explained signal is accumulated. The amount of noise generated decreases as more and more signal is explained, and as the energy in the residual receiver trace is reduced. When no coherent signal can be extracted anymore, the last residual deconvolution result is added in order to not lose any signal in the end result.

We illustrate this step using a synthetic data example where we modelled a receiver trace over an earth model containing three reflectors and seven point diffractors, using a source continuously emitting band-limited Gaussian white noise while moving. The modelled receiver trace is shown at the top of Figure 3 . It has a length of 3200s which corresponds to a streamer length of $8000 \mathrm{~m}$ and a vessel speed of $2.5 \mathrm{~m} / \mathrm{s}$. The final deconvolution result is shown at the bottom of Figure 3 together with a modelled reference earth response and the difference between the two. The energy level in the difference is very small illustrating the effectiveness of the iterative source deconvolution method. 

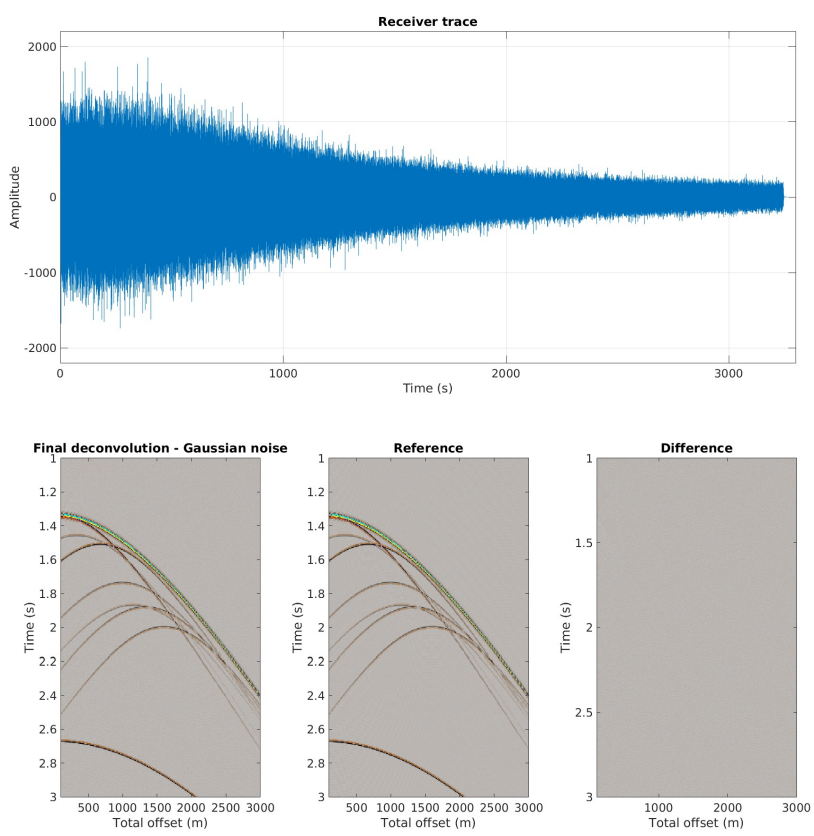

Figure 3 - Top: Simulated receiver trace in a stationary position containing more than 3000 seconds of signals received continuously. Bottom: Results from the iterative source deconvolution process shown to the left. The image in the middle shows the desired output, and the image to the right shows the difference between the deconvolution result and the desired result.

The desired signal for the continuous wavefields method is that of band-limited white noise, as this enables deconvolution of the total source wavefield from the received wavefield. A seismic source emitting bandlimited white noise does not exist. It is however possible to approach the properties of white noise by triggering individual air-guns densely with randomized time intervals. Typically, a seismic vessel tows six strings of air-guns allowing for the layout shown in Figure 4. Each string is equipped with three different volumes in a different order. The air-guns are triggered one by one cycling through the strings and the air-guns within a string in a pre-defined order. With a mean trigger time interval in the order of $300 \mathrm{~ms}$ and a suitable randomization interval, a source wavefield is generated which approaches the properties of white noise.

Since each string is set up differently, the cross-line horizontal wavenumber spectrum of the source wavefield is broad-band. This allows us to output common receiver gathers in each cross-line position with source elements resulting in a hexa-source setup. After the deconvolution, individual sources can be combined if desirable. Figure 5 shows the deconvolution result for a receiver trace modelled in the same location over the same geological model as used for Figure 3, but using the setup shown in Figure 4 with a string separation of $200 \mathrm{~m}$. The different temporal and spatial position of the diffraction events illustrates the 3D character of the simulation. The residual noise level is only slightly increased compared to the single source case simulated with white noise as the source wavefield, as shown in Figure 3.

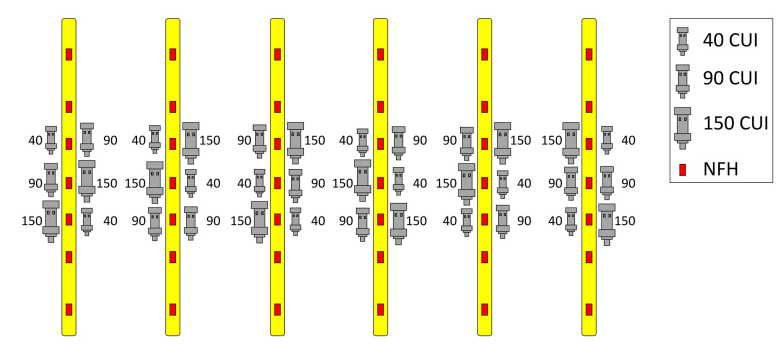

Figure 4 - Six strings with 40, 90 and 150 cubic-inch airguns on each. The configuration of the three volumes is different on each sub-array to provide additional encoding of the wavefield emitted from each string.

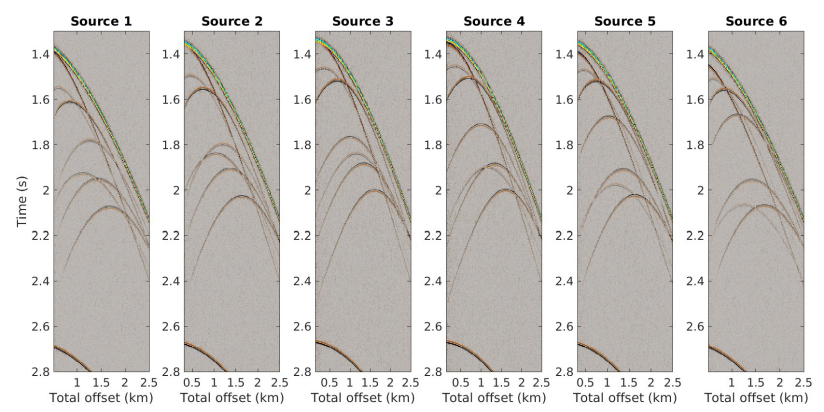

Figure 5 - Result of deconvolving the source wavefield into six common receiver gathers with earth responses extracted from one stationary receiver location based on the source configuration shown in Figure 4. The spacing between the strings of air-guns was $200 \mathrm{~m}$.

\section{Improved source side sampling}

At $4 \mathrm{~ms}$ temporal sampling interval, the offset increment in the output receiver gather from the source deconvolution is $6.25 \mathrm{~m}$, i.e. the signal is fully de-aliased. This is facilitated by the continuous source wavefield. With 12.5 $\mathrm{m}$ channel spacing on the receiver side, the receiver gathers are anti-alias protected to $12.5 \mathrm{~m}$ spacing as well to create symmetric wavenumber content on the source and receiver side in the inline direction. A shot point spacing of $12.5 \mathrm{~m}$ along the line is otherwise only achievable with very short records or blended acquisition. The proposed method therefore generates band-limited point sources with denser spatial sampling along the line.

When source elements in different cross-line positions are emitting energy simultaneously, it is possible to output common receiver gathers in each cross-line position from the iterative source deconvolution as illustrated in Figure 5 . This potentially allows great flexibility in survey design. When optimizing source and streamer positions, configurations can be found which optimally combine survey efficiency with sampling requirements. For example, the hexa-source configuration shown in Figure 6 would allow for denser crossline CMP spacing together with increased sail-line distance, i.e. increased efficiency, 
compared to the triple source configuration shown in the same Figure. At present this is a concept and has not been tested.
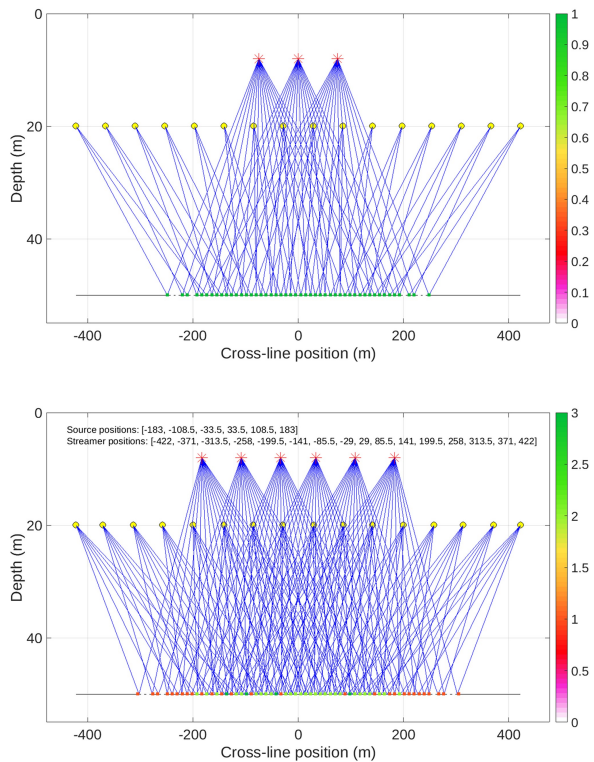

Figure 6 - Top: Towed streamer configuration with 3 sources, $75 \mathrm{~m}$ separation, and 16 streamers, $56.25 \mathrm{~m}$ separation. The nominal sail line spacing with this configuration is $450 \mathrm{~m}$. The colored squares at the reflector level shows the number of hits per bin in the cross-line direction. Bottom: A 6 source, 16 streamer, configuration determined through an optimization process. The nominal sail line separation is $550 \mathrm{~m}$ for this configuration. The source and streamer positions are listed in the upper part of the image.

\section{Reduced environmental footprint}

One of the main benefits of spreading the emitted wavefield from the sources in time is a potential reduction of sound levels. Traditionally, in the order of 30-35 airguns in a source array are activated simultaneously. By activating one air-gun at the time the peak sound pressure levels (peak SPL) are reduced significantly compared to activating a 4130 cubic-inch source array as illustrated in Figure 7. A comparison between the SEL, integrated over 10 seconds, of the standard array and the activation of individual air-guns is shown in Figure 8 for one direction from the source. We can see in that modelling study that the SEL is lower when triggering individual air-guns.

Both the peak SPL and SEL are here determined through modelling assuming wave propagation through a homogeneous medium with properties of water and a sea surface reflection coefficient of -1 . This modeling approach is widely used in permitting processes and follows the standard metrics used in the industry today (GOERTZ et al., 2013). Still, it is important to note that the SEL results presented here should be considered as a relative comparison and not an absolute measure.

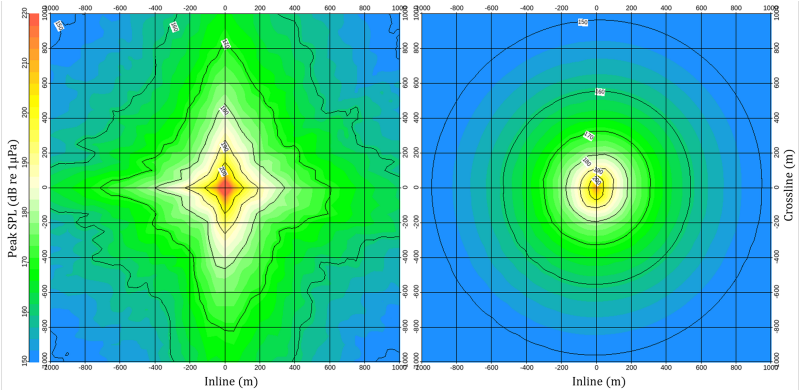

Figure 7 - Peak sound pressure levels (in $d B$ re $1 \mu P a$ ) as a function of inline and cross-line distances in meters from the geometrical center of the source at a depth of 10 $m$ (4 $m$ below the source depth) for a conventional 4130 cubic-inch array (left) and when triggering individual airguns in a near-continuous fashion (right).
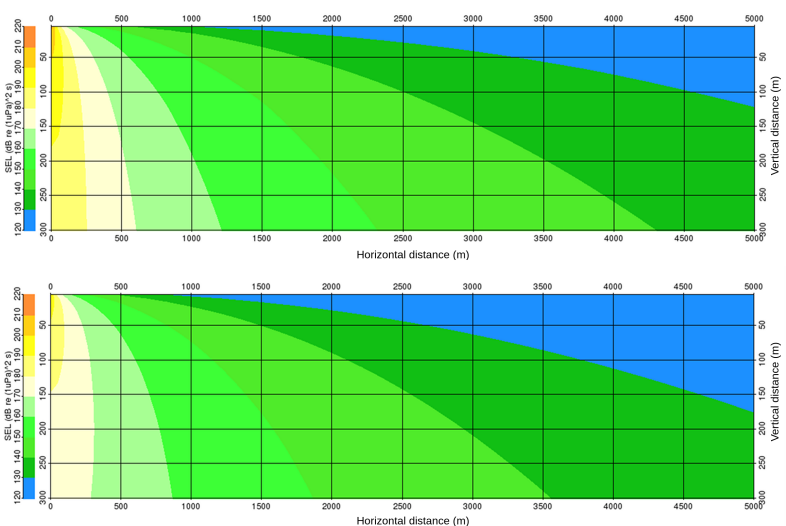

Figure 8 - Sound exposure levels (in $d B$ re $1 \mu P a 2 s$ ) as a function of distance from the source. Horizontal axis is meters from the geometrical center of the array directly aft from the vessel, while the vertical axis is depth from the sea surface in meters. The integration time is set to 10 seconds starting from the first arrival at each location. Conventional 4130 cubic-inch array (top) is compared with triggering individual air-guns in a near-continuous fashion on the bottom.

\section{Conclusions}

We have presented a novel marine seismic acquisition and processing method that utilizes continuous source and receiver side wavefields. An iterative scheme for deconvolving the source wavefield extracts the response of the earth associated with each cross-line position where source elements were deployed, because the wavefield emitted can be designed such that it is spatially white also in the cross-line direction. The typical six airgun strings towed by a seismic vessel can be configured in a way that a hexa-source setup is achieved. That potentially gives a high degree of flexibility in survey design allowing for very efficient acquisition configurations which do not sacrifice spatial sampling. 
When individual air-guns are triggered with dense, randomized time intervals, source energy is spread out in time which leads to reduced sound pressure levels when compared to standard air-gun arrays.

\section{Acknowledgments}

We thank PGS for permission to publish this paper, and Equinor and The Research Council of Norway for funding the project together with PGS.

\section{References}

ABMA, R., ROSS A., Popcorn shooting: Sparse inversion and the distribution of airgun array energy over time. 83rd Annual International Meeting, SEG, Expanded Abstracts, p. 31-35, 2013.

BEASLEY, C.J., CHAMBERS, R.E., JIANG, Z. A new look at simultaneous sources. 68th Annual International Meeting, SEG, Expanded Abstracts, p. 133-135, 1998.

BERKHOUT, A.J. Changing the mindset in seismic data acquisition. The Leading Edge, V. 27, p. 924-938, 2008.

CARLSON, D., LONG, A., SÖLLNER, W., TABTI, H., TENGHAMN, R., LUNDE, N. Increased resolution and penetration from a towed dual sensor streamer. First Break, V. 25, p. 71-77, 2007.

FRØMYR, E., CAMBOIS, G., LOYD, R., KINKEAD, J. Flam - A simultaneous source wide azimuth test. 78th Annual International Meeting, SEG, Expanded Abstracts, p. 2821-2824, 2008.

GOERTZ, A., WISLØFF, J.F., DROSSAERT, F. ALI, J. Environmental source modelling to mitigate impact on marine life. First Break, V. 31, p. 59-64, 2013.

HEGNA, S., KLÜVER, T., LIMA, J. Making the transition from discrete shot records to continuous wavefields Methodology. 80th Conference and Exhibition, EAGE, Extended Abstracts, We A10 03, 2018.

KLÜVER, T., HEGNA, S., LIMA, J. Making the transition from discrete shot records to continuous wavefields Real data application. 80th Conference and Exhibition, EAGE, Extended Abstracts, We A10 04, 2018.

MUELLER, M.B., ROBERTSSON, J.O.A., HALLIDAY, D.F. Simultaneous source separation using encoded source sequences. 76th Conference and Exhibition, EAGE, Extended Abstracts, Th ELI2 03, 2014.

ROBERTSSON, J.O.A., AMUNDSEN, L., SJØEN PEDERSEN, A. Wavefield Signal Apparition, Part I Theory. 78th Conference and Exhibition, EAGE, Extended Abstracts, We LHR2 05, 2016.

SJØEN PEDERSEN, Å., AMUNDSEN, L., ROBERTSSON, J.O.A. Wavefield Signal Apparition, Part II - Application to simultaneous sources and their separation. 78th Conference and Exhibition, EAGE, Extended Abstracts, We LHR2 06, 2016. 